\title{
Ultrathin plasmonic chiral phase plate
}

\author{
Yuri Gorodetski, ${ }^{1, *}$ Cyriaque Genet, ${ }^{2}$ and Thomas W. Ebbesen ${ }^{2}$ \\ ${ }^{1} M e c h a n i c a l$ Engineering and Mechatronics and Electrical Engineering and Electronics departments, Ariel University, Ariel 407000, Israel \\ ${ }^{2} I S I S$ and icFRC, UMR 7006, CNRS-Université de Strasbourg, 8 Allée G. Monge, 67000 Strasbourg, France \\ *Corresponding author: yurig@ariel.ac.il
}

Received 21 July 2016; revised 18 August 2016; accepted 26 August 2016; posted 26 August 2016 (Doc. ID 271979);

published 15 September 2016

\begin{abstract}
A thin free-standing gold membrane with complex plasmonic structures engraved on both sides is shown to perform as an ultrathin phase plate. Specifically, we demonstrate the generation of a far-field vortex beam propagating at a desired angle. The angular momentum of the beam is generated by the groove helicity, together with the geometric phase arising from a plasmonic spin-orbit interaction. The radial chirp of the back-side structure is used to modify the emission angle via a specific momentum matching condition. (C) 2016 Optical Society of America
\end{abstract}

OCIS codes: (240.6680) Surface plasmons; (310.6628) Subwavelength structures, nanostructures; (350.2770) Gratings.

http://dx.doi.org/10.1364/OL.41.004390

Plasmonics-based flat optics has become extremely popular due to its compactness and highly efficient light confinement provided by the evanescent nature of the surface waves [1-3]. Two-dimensional (2-D) geometry of these waves leads to miniaturizing optical elements to subwavelength volumes, providing a variety of practical applications from nanophotonic sensors $[4,5]$ to optical communication devices and photonic logic circuits [6-8]. Owing to the specific dispersion of the surface plasmons (SPs), the light can be easily coupled to a plasmonic mode and shaped through shallow corrugations on metal films [9-11]. The geometry of these corrugations with respect to the incident light provides the means to modulate the phase and the amplitude of the plasmonic field. Moreover, it has been shown that axially symmetric structures, such as bull's eyes and spirals, lead to plasmonic spin-orbit coupling due to the polarization selectivity of the light-SP coupling process [12-16]. Accordingly, the incident light can be directly coupled to plasmonic modes carrying orbital angular momentum (OAM) $[17,18]$. The OAM of a plasmonic field is manifested via its helical phase ramp of a form $\exp (i l \varphi)$, where $\varphi$ is an azimuthal coordinate, and $l$ is called the topological charge of the vortex. The total OAM of these plasmonic vortices is composed of the spin contribution (due to the handedness of the incident circular polarization) and the dynamic phase added by the spiral structure $[17,19]$.

Recently, it was shown that by using a suspended metal membrane with corrugations on both sides, interconnected by a subwavelength aperture, one can directly tailor a beam phase in the far field [15]. Specifically, it was shown that propagating vortices with spin-dependent OAM can be excited using only a $300 \mathrm{~nm}$ thick plasmonic device. The incident light was coupled to an SP vortex on one side of the membrane, propagated to the other side through a central hole and then re-emitted to the free space, with a spiral phase generated by the plasmonic structures' geometries. While a pitch of the spiral groove induced the phase helicity, the periodicity of the grooves provided a resonant in-coupling and out-coupling of light at plasmonic structures. We note that these two degrees of freedom can be manipulated independently in our structure. Namely, the vortex OAM can be controlled by the structure topology, while the way the SP mode couples to the free space can be tweaked by modifying the local periodicity of the grating.

In this Letter, we propose and experimentally demonstrate an independent manipulation of the far-field OAM and a beam steering angle by the use of a double-sided plasmonic structure. This is achieved by modifying the periodicity of the grating at the exit side as a function of the local azimuthal angle. The SP mode, launched by the frontside grating and coupled to the back side via the central hole is beamed out by a back-side grating. This grating is designed to locally ensure a momentum matching, depending on the SP wave vector orientation. This results in a collective beaming of a vortex with a desired OAM in a given off-axis direction by the use of a single ultrathin chiral plasmonic phase plate. A beam steering by means of specially designed plasmonic structures was recently presented in several works [20-24]. In contrast with these solutions, generally based on mirror symmetry breaking of the structure, our proposed scheme directly utilizes the dispersion properties of the SP waves to beam out the light at a precise angle. The ability to independently modify the structure on both sides of a thin membrane provides a flexible and simple way to design beams with abrupt phases. Finally, an external control of the emerging beam OAM is provided by an accurate choice of the polarization state of light. These functionalities might find important applications in the field of optical communication for multiplexing and demultiplexing of signals, plasmonic circuits, optical interconnects, and ultracompact optical guides.

We consider a $300 \mathrm{~nm}$ thick suspended gold membrane with spiral grooves engraved on both sides, connected via a central sub-wavelength hole $(d=400 \mathrm{~nm})$. The aim of the 
frontside structure is to couple the light into a SP mode propagating toward the hole, while the exit structure couples the SPs back to the far-field propagating light.

The general groove radial path milled on the metal surface is given in the polar $(\hat{\boldsymbol{\rho}}, \hat{\boldsymbol{\varphi}})$ basis, as $\rho_{n}=(n \Lambda+m \varphi \Lambda / 2 \pi)$, with $n$ being an integer, $\Lambda$ being the spiral grating period, and $m$ being a pitch number. When illuminated with circularly polarized light $\left(\sigma^{ \pm}=\left(E_{0}^{x} \pm i E_{0}^{y}\right) / \sqrt{2}\right)$, a properly designed surface structure excites a plasmonic vortex with the field given in the form $E_{\mathrm{SP}}^{ \pm} \propto e^{i(m \pm 1) \phi}[12,14-16]$. The OAM of the frontside mode is given via its topological charge $l_{1}=m_{1} \pm 1$ and stems from the helicity of the coupling structure and from the spin of the incident light $[13-15,17]$. Hereafter, index 1 indicates the membrane front side and index 2 stands for the exit side. The SP vortex, excited on the front side propagates through the central aperture and transfers the OAM to the backside SP mode which is then scattered to the far field by the additional structure. While the full discussion on the mechanism of the SP mode coupling to the cylindrical hole waveguide mode is given in [15], we briefly note that the hole size was optimized to maximize the SP transmission to the back surface. There, the SP mode radially diverging from the hole outcouples to the far field with an additional OAM of $l_{2}=m_{2} \pm 1$, depending on the emerging polarization state. The total topological charge of the resulting far-field beam is then given as $l=l_{1}-l_{2}$, and its values are summarized in Table 1 for each incident/emerging spin state [15].

The momentum matching condition between the free-space on-axis radiation and the SP mode is ensured by a perfectly concentric structure with a constant periodicity that equals plasmonic wavelength, $\Lambda=\lambda_{\mathrm{SP}}$. We note that by locally modifying the momentum matching condition, one can achieve an off-axis beaming in any desired direction. This can be done by properly tuning the local periodicity along the azimuthal direction.

To do this, we consider a 2-D dispersion relation of the surface mode (see Fig. 1). The cross section of the dispersion at the laser frequency $\omega=\omega_{0}$ represents the reciprocal plane of the SPs propagating on the back surface in the radial direction. The solid blue circle corresponds to the SP wave vectors given by the equation $\left(2 \pi / \lambda_{\mathrm{SP}}\right)^{2}=k_{\mathrm{SP}-x}^{2}+k_{\mathrm{SP}-\gamma}^{2}$. The light-cone (dashed line) encircles the region where the free light propagation is allowed. The blue dot represents a wave vector of the free-space beam emerging from the structure on the back side of the membrane. When the light beams out in a normal direction, its transverse wavenumber is zero which corresponds to the origin in the $k$-space. Without a loss of a generality, we suppose that the emerging beam is tilted at an angle $\theta$ in $x$ direction. Its transverse wavenumber is then given by $k_{x}=\left(2 \pi / \lambda_{0}\right) \sin (\theta)$, where $\lambda_{0}$ is the wavelength in vacuum. The momentum mismatch between the diverging plasmonic mode (a blue circle) and the beam with the desired wavenumber (a blue dot) is measured by the distance between them in

Table 1. Far-Field Summation Rules for OAM Generated through the Membrane

\begin{tabular}{ccc}
\hline & + & - \\
\hline+ & $m_{2}-m_{1}$ & $m_{2}-m_{1}+2$ \\
- & $m_{2}-m_{1}-2$ & $m_{2}-m_{1}$ \\
\hline
\end{tabular}

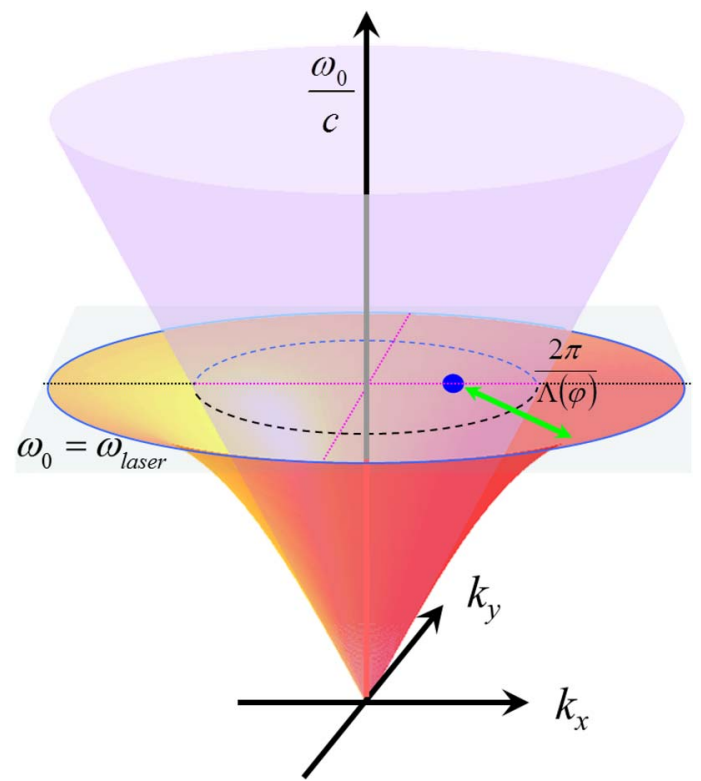

Fig. 1. Schematic representation of the SP dispersion. The blue dot represents the desired wave vector of the emerging light; the dashed circle corresponds to the plasmonic mode at the laser frequency, $\omega_{\text {laser }}$; the arrow shows the momentum mismatch to be compensated by the back-side grating period $\Lambda(\varphi)$.

the $k$ space as, $\left(k_{\mathrm{SP}-x}-k_{x}\right)^{2}+\left(k_{\mathrm{SP}-y}\right)^{2}=(2 \pi / \Lambda(\varphi))^{2}$. The substitution of the Cartesian components of the SP wavenumbers, $k_{\mathrm{SP}-x}=\left(2 \pi / \lambda_{\mathrm{SP}}\right) \cos (\varphi), k_{\mathrm{SP}-y}=\left(2 \pi / \lambda_{\mathrm{SP}}\right) \sin (\varphi)$ yields

$$
\Lambda(\varphi)=\left[\frac{1}{\lambda_{\mathrm{sp}}^{2}}+\frac{\sin ^{2} \theta}{\lambda_{0}^{2}}-\frac{2 \sin \theta \cos \varphi}{\lambda_{0} \lambda_{\mathrm{sp}}}\right]^{-\frac{1}{2}} .
$$

It is clear from this relation that the variation of the period as a function of the azimuthal angle provides an additional degree of freedom and does not interfere with the mechanism of a spiral phase generation achieved by the variation of the groove radii. We demonstrate this by fabricating a $300 \mathrm{~nm}$ thick membrane consisting of a bull's eye structure $\left(m_{1}=0\right)$ on the front side and a right spiral $\left(m_{2}=1\right)$ structure with a space-variant period on the back side (BE-R). The desired tilt angle of the transmitted beam is chosen to be $10^{\circ}$.

This structure is fabricated on a suspended thin ( $h \sim 300 \mathrm{~nm})$ metallic membrane evaporated on a metal film over a poly(vinyl formal) resin supported by a transmission electron microscopy copper grid. After evaporation, the resin is removed by using a focused ion beam (FIB), leaving a freely suspended gold film. Plasmonic structures are milled using FIB lithography on both sides of the membrane around a unique central cylindrical aperture acting as the sole transmissive element of the whole device. The diameter of the hole is chosen to be $400 \mathrm{~nm}$ to allow a transmission of the fundamental $\mathrm{TE}_{11}$ plasmonic mode. The center alignment of the structure on the back side is ensured by the central hole that is milled through the membrane.

The membrane is illuminated by a single-mode fiber pigtailed laser diode at $\lambda_{0}=785 \mathrm{~nm}$ using a setup shown in Fig. 2(a) (see the caption for the details). The depth of the grooves $(30 \mathrm{~nm})$ is fixed to be smaller than the skin depth $\left(\sim 70 \mathrm{~nm}\right.$ at $\left.\lambda_{0}\right)$ to avoid direct light transmission through 

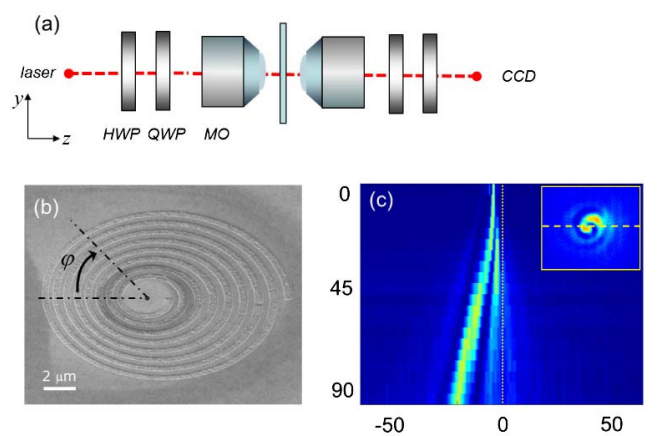

Fig. 2. (a) Schematics of the experimental setup. The polarization of the beam is set by transmitting a linearly polarized Gaussian laser beam through a half-wave plate and a quarter-wave plate (QWP). The selection of a specific circular polarization is obtained by an additional set of a QWP and a polarizer with a relative orientation of $\pm 45^{\circ}$. Microscope objectives were used to illuminate and to image the structure. (b) SEM image of the back-side structure with a period dependence on $\varphi$. (c) Evolution of the beam's cross section upon propagation along the $z$ axis. The cross section of the images at each distance were taken along a central line (see the inset). The dimensions are given in microns.

the membrane. A scanning-electron microscope (SEM) image of the structure is shown in Fig. 2(b).

As expected from the double-sided structure [15], a free propagating vortex is produced behind the membrane whose field distribution is given in a vector form as $\mathbf{E}_{\text {out }} \propto \exp \left[i\left(m_{1}-\right.\right.$ $\left.\left.m_{2} \mp 1\right) \varphi\right](\cos (\varphi), \sin (\varphi))^{T}$ with the incident state of circular polarization represented by $(+)$ for the right-handed and (-) for the left-handed. The resulting radially polarized vector field can be decomposed into circularly polarized vortex components $[15,17]$. These vortex beams propagate behind the membrane and may interfere with the light, directly transmitted through the hole. Interestingly, the interference contrast can be controlled using the incident-emerging polarization state combination $(i, j)$, where $j$ is the incident state and $i$ is the emerging state. We are particularly interested in the two special cases of $(+,+)$ [direct transmission is allowed] and $(-,+)$ [full extinction of direct transmission]. The explicitly calculated azimuthal part of the fields corresponding to these combinations is given as $E_{-+} \propto e^{3 i \varphi}$ and $E_{++} \propto e^{-i \varphi}$. In terms of the angular momentum, these fields represent vortices with an OAM $l_{-+}=+3$ and $l_{++}=-1$. In Fig. 2 (c) we show the cross section of the intensity distribution behind the membrane measured along the $\mathrm{z}$ axis for the latter case. We note a well pronounced beam tilt with respect to the propagation direction.

In Fig. 3, the intensity distributions of the transmitted beams are shown for the $( \pm,+)$ situations as a function of the propagation distance. The spiral fringe arising from the interference with the direct transmission is clearly visible in Fig. 3(a), as compared to the "doughnut" intensity distribution in Fig. 3(b) expected from a pure optical vortex. This spiral interference pattern is very useful for validating the resultant OAM of the free propagating light beam. In particular, as we showed in [15], a spiral fringe with one wing unwrapping in a clockwise direction, visible in Fig. 3(b) corresponds to the topological charge of -1 . The measured tilt of the transmitted beam was found to be $9.98^{\circ}$ which perfectly corresponds to the structure design. This demonstrates that by modifying both the

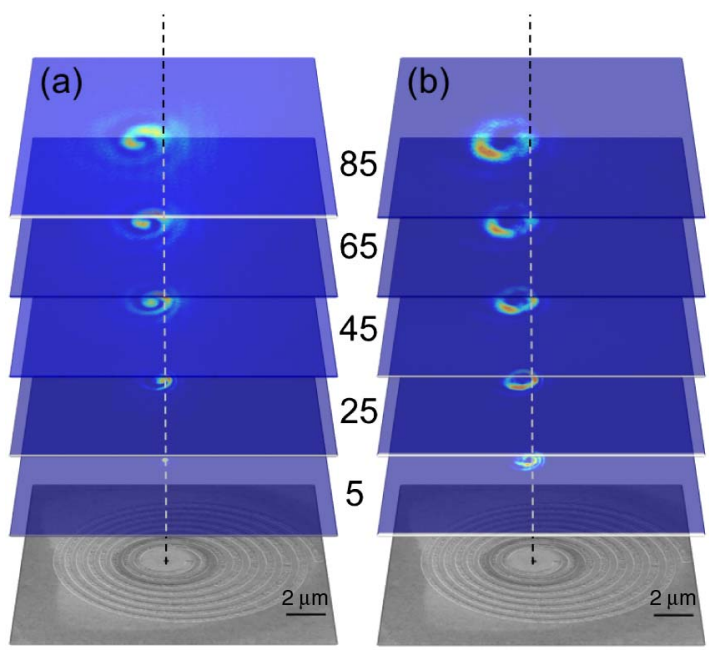

Fig. 3. Propagation evolution of the beam generated by a $B E-R$ structure in a polarization configuration $(\mathrm{a})(+,+)$ and $(\mathrm{b})(-,+)$. The distances are given in microns.

azimuthal phase lag of SPs and the periodicity of the structure, one can separately control the helicity of the beam and the propagation direction. Note that when the direct transmission is fully filtered out by the analyzer, no zero order is obtained. In this case, a membrane with a modified structure on the back side acts as a pure phase modulator in contrast with diffractive elements or holographic plates. Moreover, by modifying the front side in a similar way (not shown here), one can control the resonant incident angle and even mimic negative refraction.

Here we have shown that a suspended thin golden membrane with nanostructures on both sides can perform as an ultrathin phase element. Light-plasmon interaction leads to an abrupt phase change of the transmitted beam and allows us to modify the beam's propagation in a subwavelength thickness. This scheme can be extended to other functionalities by applying more complex phase modifications on both sides of the membrane. We believe that our experiment can inspire various practical applications in nanophotonic multiplexing, optics communication devices, microscopy, and flat optics.

Funding. ANR Equipex Union (ANR-10-EQPX-52-01); Labex NIE Projects (ANR-11-LABX-0058 NIE).

\section{REFERENCES}

1. N. Yu and F. Capasso, Nat. Mater. 13, 139 (2014).

2. G. Zheng, H. Mühlenbernd, M. Kenney, G. Li, T. Zentgraf, and S. Zhang, Nat. Nanotechnol. 10, 308 (2015).

3. A. V. Kildishev, A. Boltasseva, and V. M. Shalaev, Science 339 , 1232009 (2013).

4. J. N. Anker, W. P. Hall, O. Lyandres, N. C. Shah, J. Zhao, and R. P. Van Duyne, Nat. Mater. 7, 442 (2008).

5. M. E. Stewart, C. R. Anderton, L. B. Thompson, J. Maria, S. K. Gray, J. A. Rogers, and R. G. Nuzzo, Chem. Rev. 108, 494 (2008).

6. J. Leuthold, C. Hoessbacher, S. Muehlbrandt, A. Melikyan, M. Kohl, C. Koos, W. Freude, V. Dolores-Calzadilla, M. Smit, I. Suarez, J. Martínez-Pastor, E. Fitrakis, and I. Tomkos, Opt. Photon. News 24 (5), 28 (2013).

7. S. I. Bozhevolnyi, V. S. Volkov, E. Devaux, and T. W. Ebbesen, Phys. Rev. Lett. 95, 046802 (2005).

8. N. Engheta, Science 317, 1698 (2007).

9. W. L. Barnes, A. Dereux, and T. W. Ebbesen, Nature 424, 824 (2003). 
10. H. F. Ghaemi, T. Thio, D. E. Grupp, T. W. Ebbesen, and H. J. Lezec, Phys. Rev. B 58, 6779 (1998).

11. F. López-Tejeira, S. G. Rodrigo, L. Martn-Moreno, F. J. Garca-Vidal, E. Devaux, T. W. Ebbesen, J. R. Krenn, I. Radko, S. I. Bozhevolnyi, M. U. González, J. C. Weeber, and A. Dereux, Nat. Phys. 3, 324 (2007).

12. T. Ohno and S. Miyanishi, Opt. Express 14, 6285 (2006).

13. K. Y. Bliokh, Y. Gorodetski, V. Kleiner, and E. Hasman, Phys. Rev. Lett. 101, 030404 (2008).

14. Y. Gorodetski, A. Niv, V. Kleiner, and E. Hasman, Phys. Rev. Lett. 101, 043903 (2008).

15. Y. Gorodetski, A. Drezet, C. Genet, and T. W. Ebbesen, Phys. Rev. Lett. 110, 203906 (2013).

16. Q. Jiang, A. Pham, M. Berthel, S. Huant, J. Bellessa, C. Genet, and A. Drezet, ACS Photon. 3, 1116 (2016).
17. Y. Gorodetski, N. Shitrit, I. Bretner, V. Kleiner, and E. Hasman, Nano Lett. 9, 3016 (2009).

18. Y. Gorodetski, S. Nechayev, V. Kleiner, and E. Hasman, Phys. Rev. B 82, 125433 (2010).

19. L. Allen, S. M. Barnett, and M. J. Padgett, Optical Angular Momentum (CRC Press, 2003).

20. H. Caglayan, I. Bulu, and E. Ozbay, J. Phys. D 42, 045105 (2009).

21. E.-Y. Song, H. Kim, and B. Lee, in Frontiers in Optics 2013 (Optical Society of America, 2013), paper JW3A.28.

22. Y. C. Jun, K. C. Huang, and M. L. Brongersma, Nat. Commun. 2, 283 (2011).

23. J. DiMaria, E. Dimakis, T. D. Moustakas, and R. Paiella, Appl. Phys. Lett. 103, 251108 (2013).

24. H. Kim, J. Park, and B. Lee, Opt. Lett. 34, 2569 (2009). 